\title{
MANUFACTURERS' CORNER
}

\section{Design and performance of the CM20 FEG field emission TEM}

\author{
Max T. Otten, Peter M. Mul and Marc J.C. de Jong \\ Philips Electron Optics, Building AAE, 5600 MD Eindhoven, The Netherlands
}

\section{Introduction.}

When a Scanning Transmission Electron Microscope (TEM/STEM) is equipped with a thermionic electron source, its performance is limited due to poor coherence and low probe current in small spots. These limitations affect high-resolution imaging, microdiffraction, scanning imaging, as well as EDX and EELS microanalysis. Considerable improvements are provided by a Field Emission Gun (FEG), but the conventional design (as exemplified by - but not restricted to - the first commercially available Field Emission TEM, the Philips EM 400, introduced in 1977) has a number of serious drawbacks, including low maximum current, poor source stability and complicated use. For the first time now it has become possible to combine the high performance of a FEG with the many advantages of a thermionic emitter in a single instrument: the Philips CM20 FEG microscope. This paper describes the design and performance of this instrument.

\section{The field emission gun.}

The main advantages of thermionic emitters, tungsten and $\mathrm{LaB}_{6}$, are their high maximum currents (providing the high intensities needed for observation at low magnifications and dark-field techniques), ease of control and thus ease of use, low cost and relaxed vacuum requirements. Thermionic emitters suffer from low brightness, a parameter that describes the total electron emission from an emitter per unit area and unit angle. For thermionic emitters the total electron emission is high, but it comes from a large area, with $\mathrm{LaB}_{6}$ being somewhat better than tungsten. Neither comes close, however, to a FEG, which has high emission from an area that is about a factor 1000 smaller. Typical brightness values are $10^{5} \mathrm{~A} / \mathrm{cm}^{2}$ srad for tungsten, $10^{6} \mathrm{~A} / \mathrm{cm}^{2}$ srad for $\mathrm{LaB}_{6}$ and $10^{8}-10^{9} \mathrm{~A} / \mathrm{cm}^{2}$ srad for a FEG.

The effect of brightness is most noticeable in small-probe techniques. In the case of a tungsten filament it is in principle possible to obtain a very small probe $(\sim 1 \mathrm{~nm})$, but the amount of current is infinitesimal (2000 to $10000 \mathrm{x}$ less than for a FEG). A FEG therefore offers significantly better performance for small-probe techniques such as microdiffraction, scanning imaging 
and microanalysis. Another effect of high brightness is a strong improvement in the spatial and temporal coherence, due to the small source size and energy spread of the FEG, giving profound improvements for high-resolution TEM imaging.

Three types of FEG exist: the Cold Field Emission Gun (CFEG), Thermally assisted Cold Field Emission Gun (TCFEG), and the Schottky Field Emission Gun (Schottky FEG). The TCFEG is simply a heated CFEG and is very similar in overall performance to the CFEG (except for a little better stability at the expense of a higher energy spread) and will be considered here equal to the CFEG. Based on long experience with TEM Field Emission, Philips Electron Optics has chosen the Schottky FEG for the CM20. The reasons for this choice are summarised below and are considered in detail by Mul et al. [1].

In some aspects the Schottky FEG is equal to the CFEG and TCFEG, while in others it outperforms the other two FEGs. The advantages of the Schottky FEG make it ideal for operation on a TEM/STEM system:

- the brightness of the Schottky FEG is similar to that of the CFEG and TCFEG, thus giving the same performance in small-probe applications;

- the source size of the Schottky FEG is larger than that of the CFEG/TCFEG, providing high maximum currents (high intensity and thus good visibility at lower magnifications and in darkfield images) and giving a large isoplanatic patch (see below) which is important for highresolution imaging;

- the emission from the Schottky FEG is very stable, making it ideal for scanning applications (noise below $0.5 \%$ as opposed to $5-10 \%$ for a CFEG);

- the Schottky FEG is robust, with considerably relaxed requirements in comparison with a CFEG with regard to vacuum and shielding of external stray fields, and a high degree of immunity to high-tension flash-overs;

- the Schottky FEG is easy to use, so that no valuable instrument time is lost in setting up (no need for time-consuming flashing as with the CFEG).

\section{Design.}

Long experience with FEG TEMs has shown that for a modern FEG TEM ease of use is of prime importance. Few institutions can afford a dedicated FEG microscope sitting idle because too few operators can use it. Neither should time on the instrument be wasted by optimising the gun (typically $25 \%$ of the time on a CFEG instrument is lost this way). The latter is ensured by the Schottky FEG of the CM20. The ease of use is achieved because the instrument was designed in such a way that the FEG forms an integral part of the CM microscope, making operation of the CM20 FEG very similar to that of a CM instrument equipped with a thermionic emitter.

The vacuum requirements of a FEG are more stringent than those for a thermionic emitter, although the Schottky emitter will perform at vacuum pressures much higher than those tolerated by a CFEG. The overall design principle of the vacuum system of the CM20 FEG has been to reduce the number of vacuum elements and to avoid the brute-force method (that is, compensate poor vacuum design with a lot of vacuum pumps). Or, to express it in seafaring terms: it is better to have a well-caulked boat than a large bailer.

The CM20 FEG vacuum system forms an extension of the basic CM microscope system (Fig. 1). To the two-stage differential vacuum between the projection chamber and column, two more differential stages have been added, one between the column and the accelerator, and the other between the accelerator and the emitter. The position of an ion-getter pump (IGP 3 ) directly above the emitter itself ensures efficient pumping and at the same time provides a fast and accurate monitoring point for the vacuum, thereby enhancing the safety of the system. 


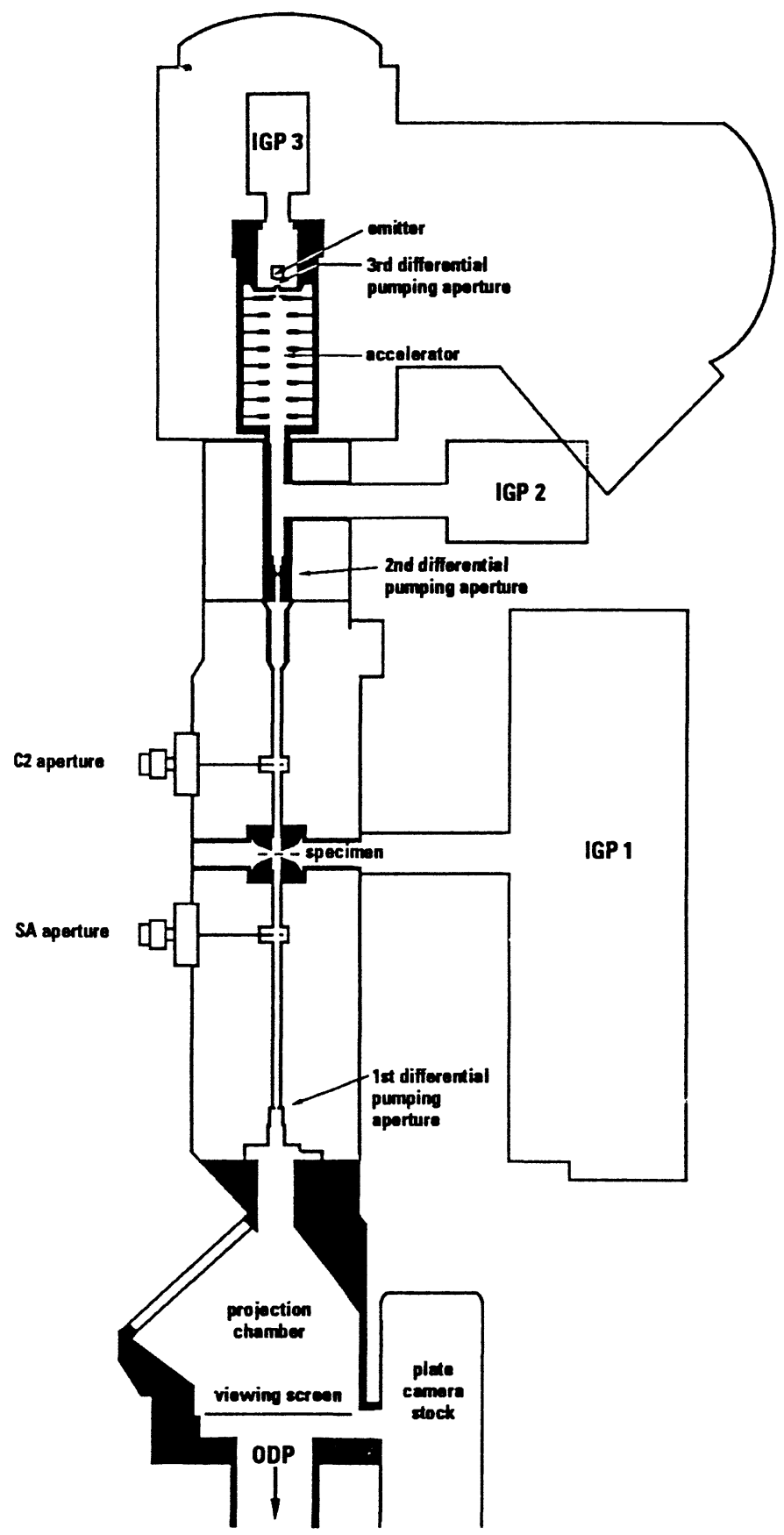

Fig. 1. - The vacuum system of the CM20 FEG contains four differential vacuum levels: 1) the projection chamber pumped by the oil-diffusion pump (ODP); 2) the column pumped to $10^{-7}$ torr by the ion-getter pump IGP 1 ; 3) the intermediate block and accelerator pumped to $10^{-8}$ torr by ion-getter pump IGP 2 ; and 4) the emitter pumped to $10^{-9}$ to $10^{-10}$ torr by ion-getter pump IGP 3 . 
The field emitter is protected by a number of safeguards such as vacuum interlocks and monitoring points, as well as automated operating procedures embedded in the CM software. The success of the protection can be inferred from the following:

- the first non-prototype instrument has been running with the same emitter for more than 1 year, during which the performance has changed by no more than a factor two;

- no emitter failure has occurred, even at voltages much higher than those achieved during normal operation (on the first $300 \mathrm{kV}$ FEG built recently voltages over $350 \mathrm{kV}$ have been achieved without causing emitter failure).

The gun of the CM20 FEG is controlled by a dedicated FEG microprocessor which communicates with the CM software and with the FEG electronics which are mounted directly behind the gun.

There are three ways of starting up the FEG: 1) the regular start (each morning) which goes from standby to operate in about 30 seconds; 2) a warm start (23 minutes); 3 ) a cold start (about 1.5 hours). The warm and cold starts are fully automated procedures [1], which are used only when the emitter was switched off altogether, with the cold start used when the emitter was off for a long period (more than 1 day).

The microscope is normally left in standby mode when not in use. The emitter heating current is reduced slightly and the extraction voltage set at a low value. Switching from standby to operate immediately increases the filament heating. The extraction voltage can be set to the desired value. Emission is stable almost from the moment of reaching the extraction voltage required. A few percent change in emission is seen during the first five minutes. After that the gun has reached its normal stability, with noise typically below $0.5 \%$ and drift $1 \%$ per hour (upward, not downward).
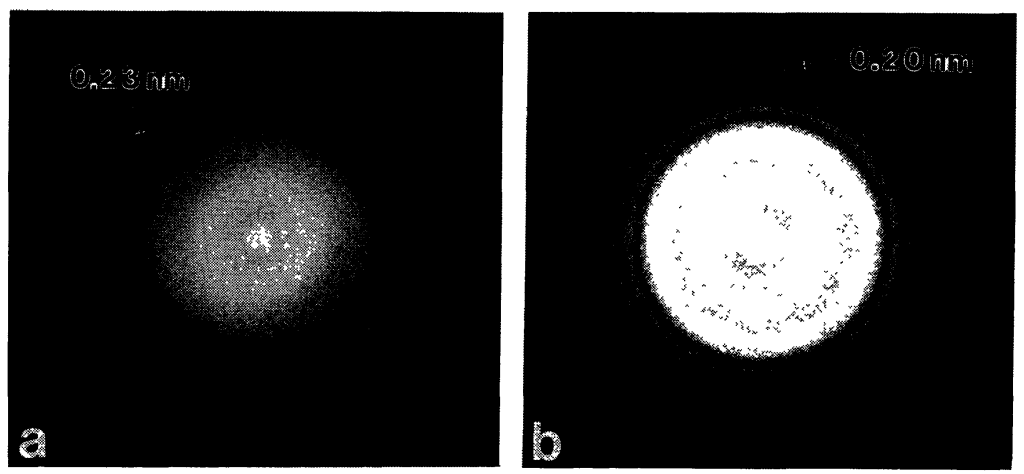

Fig. 2. - Optical diffractograms from amorphous germanium with gold islands near Scherzer defocus, taken with the CM20-TWIN in (a) $\mathrm{LaB}_{6}$ and (b) FEG versions (point resolution for both $0.27 \mathrm{~nm}$ ). The contrast transfer is improved considerably on the CM20 FEG in comparison with its $\mathrm{LaB}_{6}$ equivalent.

\section{Performance.}

High-RESOLUTION TEM IMAGING. - The point resolution of the TEM, defined as the first zero crossing of the Phase Contrast Transfer Function (PCTF) at Scherzer focus, is limited by the wavelength of the electrons and the $C_{\mathrm{S}}$ value of the objective lens. Detail well beyond the point resolution can be resolved in the region where the PCTF oscillates, determined by the temporal and spatial coherence of the microscope. The temporal coherence is defined by the chromatic aber- 




Fig. 3. - High-resolution image of the interface between GaAs and Si, taken with the CM20 FEGSuperTWIN and showing an abundance of small spacings (see also inset optical diffractogram). Specimen courtesy of Dr. B. Kabius, Kernforschungsanlage Jülich, Germany.

ration $C_{\mathrm{C}}$ of the objective lens, the energy spread $\Delta E$ of the electron source and the stability of the high tension. The spatial coherence is the degree of perfection of parallel illumination on the specimen. Both temporal and spatial coherence are much better with a FEG, giving a strong improvement in information transfer to higher frequencies (smaller image spacings). The improvement of the contrast transfer for higher frequencies can be measured experimentally with diffractograms of an amorphous film (Fig. 2) and observed in high-resolution images (Fig. 3). The contrast transfer plays an important role not only in materials science but in life science as well, where the best high-resolution images have been obtained so far (Fig. 4). The CM20 FEG is also ideal [2] for recording high-resolution images of proteins with the spot-scan technique [3-5].

Another performance indicator of high-resolution imaging on a FEG TEM is the size of the socalled isoplanatic patch, the central area in the image without significant lens aberrations. High- 


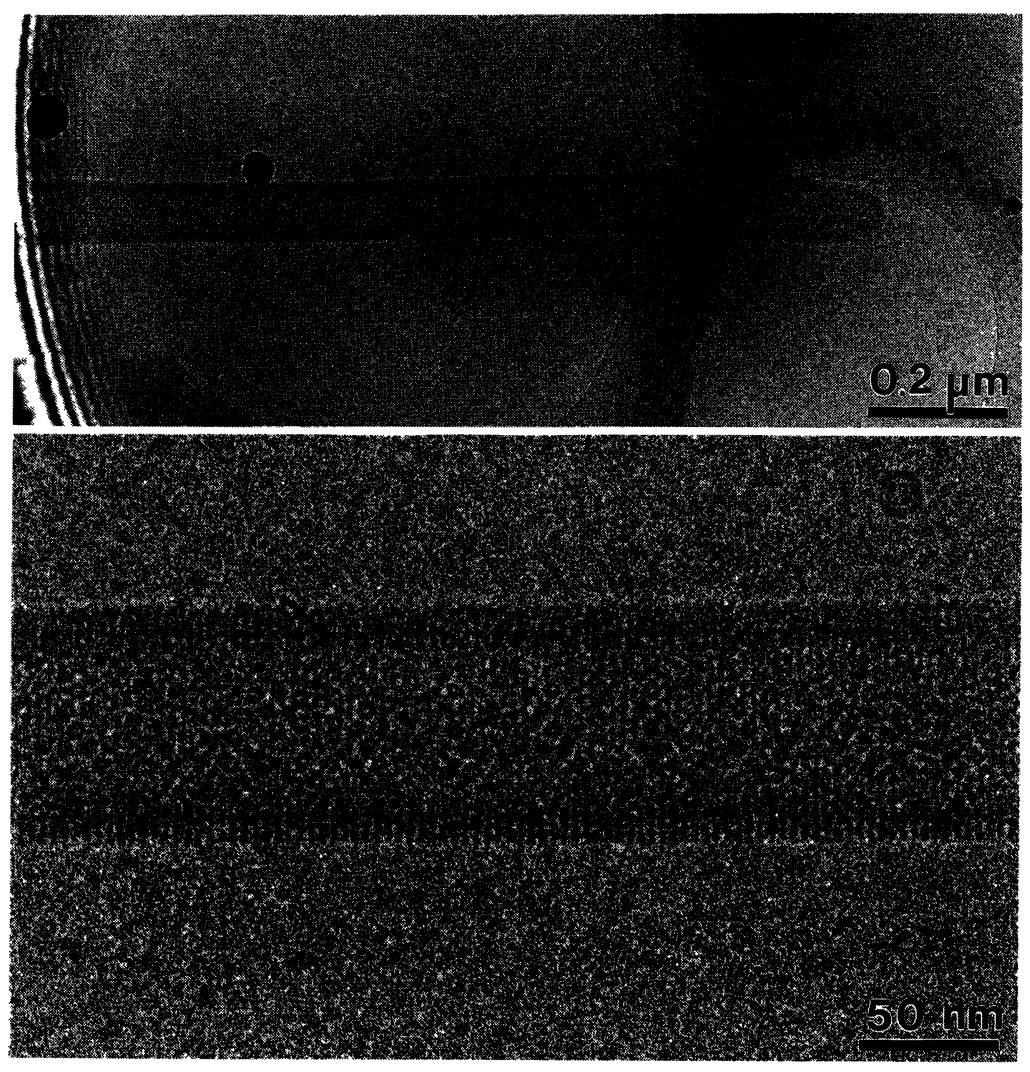

Fig. 4. - High-resolution low-dose image of acetylcholine receptor channels in a tubular vesicle from the postsynaptic membrane of a torpedo ray shows exceptionally good resolution and structure preservation. Image taken on the CM20 FEG-TWIN with the Low Dose mode at cryo temperatures. Image courtesy of Dr. N. Unwin, Medical Research Council, Cambridge, UK.

resolution imaging is based on the premise that the illuminating and imaging conditions are the same across the whole field of view. While this is generally true for an instrument equipped with a $\mathrm{LaB}_{6}$ emitter, it is not necessarily the case for one equipped with a FEG. In the latter case, the isoplanatic patch can be severely restricted to only a few tens of nanometres [6], far less than the total size of the micrograph. The area outside the isoplanatic patch is too much affected by aberrations and therefore useless.

In the case of a Schottky emitter, as used on the CM20 FEG, the isoplanatic patch (Fig. 5) is about a factor ten larger than that for a CFEG due to the Schottky's larger source size and is therefore not a limiting factor.

NANODIFFRACTION. - The Field Emission source makes it possible to obtain high-intensity probes down to about $1 \mathrm{~nm}$, while at the same time retaining small convergence angles. These small spot sizes provide diffraction information down to a very fine scale (hence the term "nanodiffraction" instead of "microdiffraction"). An example of the spatial and angular resolution obtainable with nanodiffraction on microtwins in a small gold crystal is shown in figure 6. 


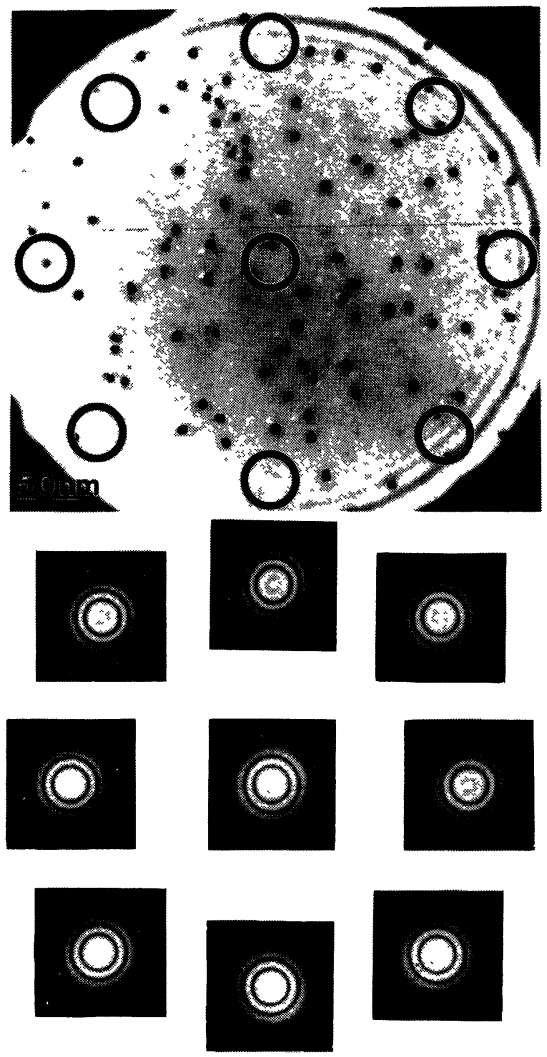

Fig. 5. - High-resolution image of amorphous germanium with gold islands, showing the whole illuminated area. The similarity of the optical diffractograms recorded in the centre and at the edges of the illuminated area demonstrates that the isoplanatic patch exceeds the illuminated area and is therefore not a problem, in contrast with the strong effects noted for a CFEG [6].

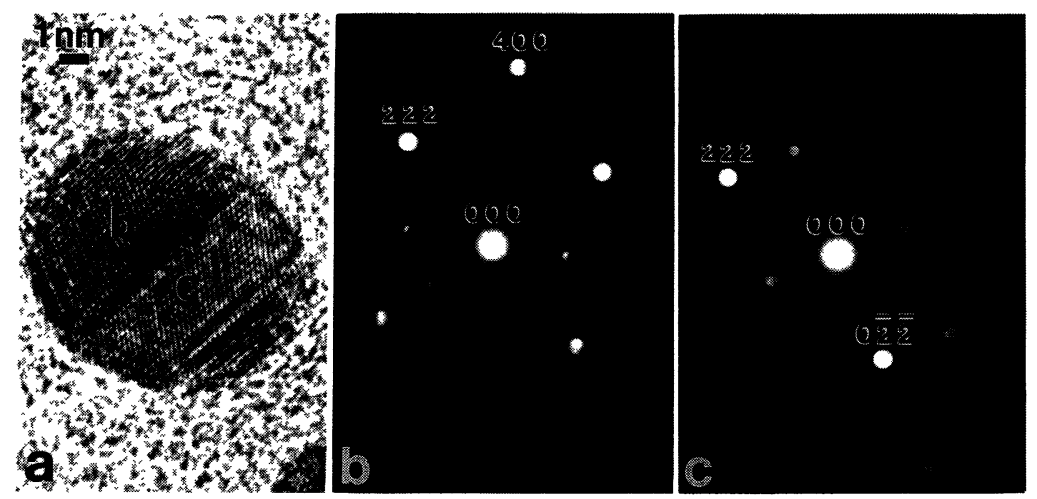

Fig. 6. - Nanodiffraction patterns (b, c) from a twin in a small gold particle (a) demonstrate the small probe size in combination with small convergence angle obtained with a CM20 FEG. 
EDX MICROANALYSIS. - A major concern in X-ray microanalysis on the TEM is the "cleanliness" of the system with regard to the "in-hole" count, which determines the proportion of signal detected from the area analysed to the amount of signal generated outside that area by hard X-rays and uncollimated electrons. The high degree of cleanliness of the CM20 FEG is demonstrated by specimen and hole-count spectra obtained from a test specimen consisting of a $100 \mathrm{~nm} \mathrm{Cr}$ film on a Cu grid (Fig. 7). The results, similar to those obtained for "real" specimens, indicate that for any element present in the specimen the X-ray signal detected with the beam placed in a hole is below $0.1 \%$ of the signal with the beam on the specimen.

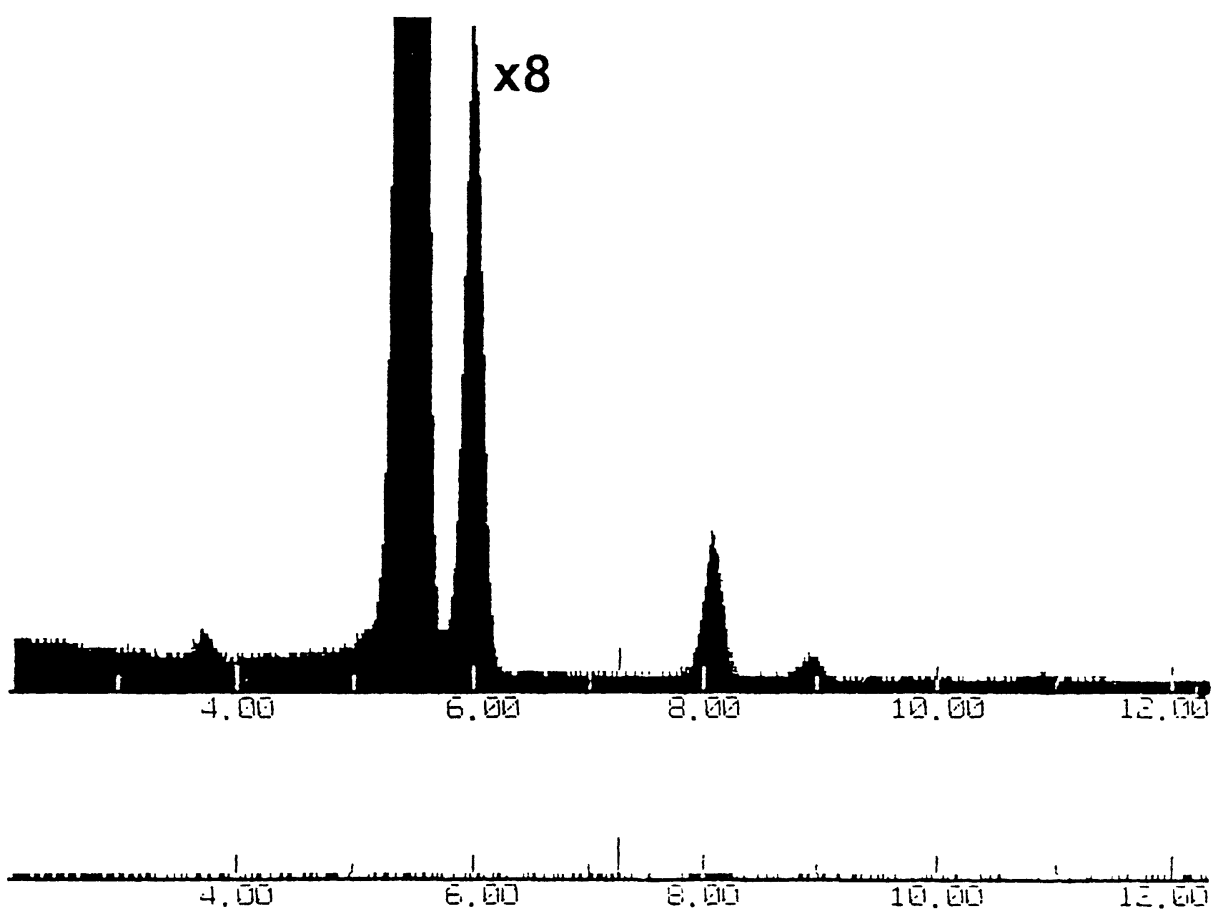

Fig. 7. - X-ray spectra, taken with a $1 \mathrm{~nm}$ probe on the CM20 FEG-TWIN, from (top) a $\mathrm{Cr}$ foil on a Cu grid and (bottom) a hole in the same specimen. The two spectra are shown at the same vertical scale, expanded $8 \mathrm{x}$. The absence of any $\mathrm{Cr}$ peak and the small $\mathrm{Cu}$ peak demonstrate the excellent "in-hole" performance of the CM20 FEG.

The performance of the CM20 FEG is crucial for understanding many important phenomena in materials, with lateral dimensions of about $1 \mathrm{~nm}$. Examples are grain or subgrain boundaries and precipitates such as Guinier-Preston zones. Analysis of such thin features is simply not feasible with $\mathrm{a} \mathrm{LaB}_{6}$ emitter due to the low signal and consequent long collection times. The FEG realises about $100 \mathrm{x}$ more current in a $1 \mathrm{~nm}$ probe than a $\mathrm{LaB}_{6}$ system and thereby makes analysis possible. An example of analysis on a small scale is provided by Guinier-Preston zones in an aluminium-copper alloy. Although one can presume that the Guinier-Preston zones have higher copper content than the matrix, to date this had not been demonstrated (Prof. B. Jouffrey, pers. comm.). With the CM20 FEG it could be demonstrated that the Guinier-Preston zones indeed contain more copper than their matrix (Fig. 8). 


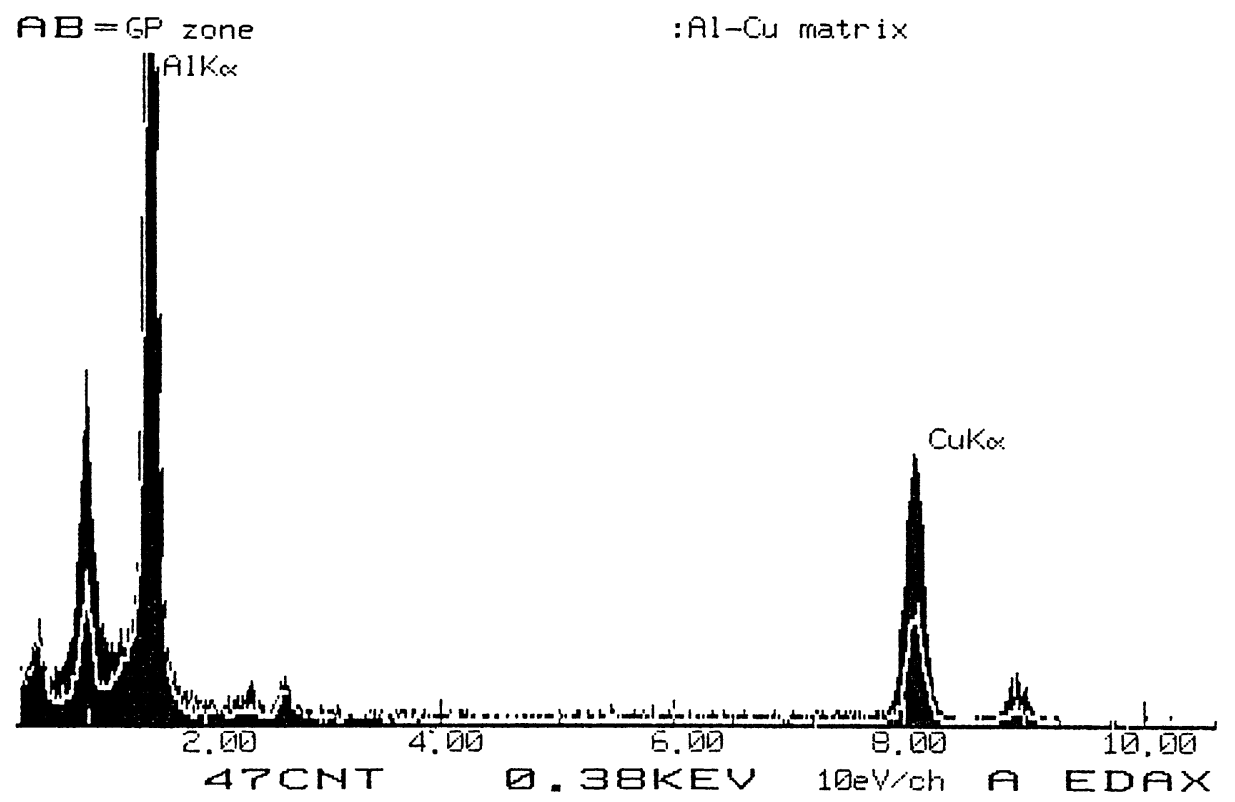

Fig. 8. - X-ray spectra of a Guinier-Preston zone (solid spectrum) and its matrix (line spectrum) in aluminium-copper alloy, demonstrating that the Guinier-Preston zone is enriched in copper. The spectra are normalised to the height of the Al peak, which is shown 2x expanded. Specimen courtesy of Prof. B. Jouffrey, Ecole Centrale Paris, France.

SCANNING IMAGING. - The scanning techniques on the TEM make efficient use of all signals generated (bright-field, dark-field and high-angle dark-field electrons, secondary electrons, backscattered electrons, $\mathrm{X}$ rays and energy-loss electrons). On microscopes equipped with a thermionic emitter, the scanning technique is limited due to large probe size (limiting the resolution) and low signal intensity (limiting visibility due to noise). The CM20 FEG microscope, with its small, highintensity probes and high stability of emission provides an ideal environment for high-performance scanning imaging.

An example comes from biology where molecules in tissue are localised by immunocytochemistry, with small gold particles (called immuno-gold labels) attached to the molecules. In recent years the size of these gold particles has been reduced to $\sim 1 \mathrm{~nm}$ in order to improve the efficiency of labelling the targeted molecules. Such ultra-small gold particles are almost invisible in the TEM image, but by using High-Angle Annular Dark-Field (HAADF) imaging $[7,8]$ they can be resolved reliably on the CM20 FEG (Fig. 9).

\section{Conclusions.}

All high-performance techniques on the TEM/STEM benefit substantially from a FEG, with major improvements in high-resolution TEM imaging, nanodiffraction, scanning imaging and microanalysis. Not all types of FEGs are suitable for the TEM, however. The two types of cold field emitters (CFEG and TCFEG) suffer from low maximum currents (dim images at low magnifications), a small isoplanatic patch and complicated use. The Schottky FEG used on the CM20 FEG does not have these disadvantages and provides therefore the ideal electron source for highperformance as well as "standard" TEM/STEM techniques. 


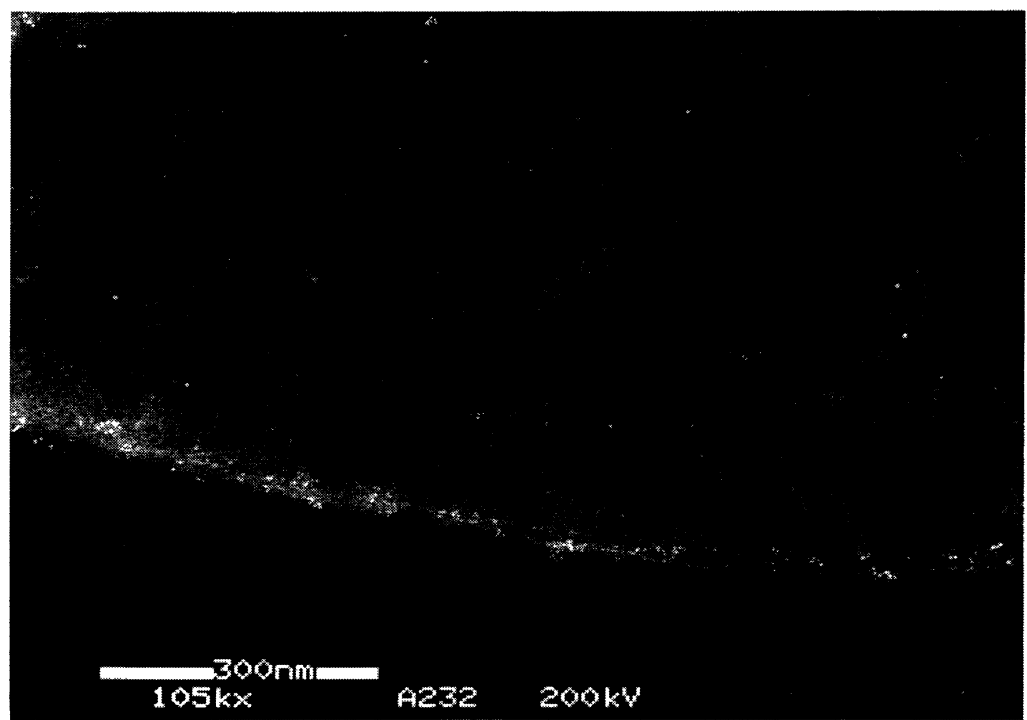

Fig. 9. - STEM High-Angle Annular Dark-Field image from a bacterium with ultra-small $(\sim 1 \mathrm{~nm})$ gold particles that are well resolved with high contrast. Specimen courtesy of Dr. Y.D. Stierhof, Max-PlanckInstitut für Biologie, University of Tübingen, Germany.

\section{References}

[1] MUl P.M., BORMans B.J.H. and OTTEN M.T., Philips Electron Optics Bull. 130 (1991) 53-62.

[2] OTTEN M.T. and de JONG M.J.C., Beitr. Elektronenmikroskop. Direktabb. Oberfl 24 (1991) 105-113.

[3] DOWNING K.H. and GLAESER R.M., Ultramicroscopy 20 (1986) 269-278.

[4] BULlOUGH P. and HENDERSON R., Ultramicroscopy 21 (1987) 223-230.

[5] DOWNING K.H., Science 251 (1991) 53-59.

[6] HANSZEN K.J., LAUER R. and ADE G., Ultramicroscopy 16 (1985) 47-57.

[7] OTTEN M.T., J. Electron Microscopy Technique 17 (1991) 221-230.

[8] OTTEN M.T., STENZEL D.J. and COUSENS D.R., Australian EM Newsletter 27 (1990) 10-16. 